\title{
Analysis of saccular aneurysms in the Barrow Ruptured Aneurysm Trial
}

\author{
Robert F. Spetzler, MD,1 Joseph M. Zabramski, MD,1 Cameron G. McDougall, MD,1 \\ Felipe C. Albuquerque, MD, ${ }^{1}$ Nancy K. Hills, PhD, ${ }^{3}$ Robert C. Wallace, MD, ${ }^{2}$ and Peter Nakaji, MD ${ }^{1}$
}

Departments of ${ }^{1}$ Neurosurgery and ${ }^{2}$ Neuroradiology, Barrow Neurological Institute, St. Joseph's Hospital and Medical Center, Phoenix, Arizona; and ${ }^{3}$ Department of Neurology, University of California, San Francisco, California

\begin{abstract}
OBJECTIVE The Barrow Ruptured Aneurysm Trial (BRAT) is a prospective, randomized trial in which treatment with clipping was compared to treatment with coil embolization. Patients were randomized to treatment on presentation with any nontraumatic subarachnoid hemorrhage (SAH). Because all other randomized trials comparing these 2 types of treatments have been limited to saccular aneurysms, the authors analyzed the current BRAT data for this subgroup of lesions.
\end{abstract}

METHODS The primary BRAT analysis included all sources of SAH: nonaneurysmal lesions; saccular, blister, fusiform, and dissecting aneurysms; and SAHs from an aneurysm associated with either an arteriovenous malformation or a fistula. In this post hoc review, the outcomes for the subgroup of patients with saccular aneurysms were further analyzed by type of treatment. The extent of aneurysm obliteration was adjudicated by an independent neuroradiologist not involved in treatment.

RESULTS Of the 471 patients enrolled in the BRAT, $362(77 \%)$ had an SAH from a saccular aneurysm. Patients with saccular aneurysms were assigned equally to the clipping and the coiling cohorts (181 each). In each cohort, 3 patients died before treatment and 178 were treated. Of the 178 clip-assigned patients with saccular aneurysms, 1 (1\%) was crossed over to coiling, and $64(36 \%)$ of the 178 coil-assigned patients were crossed over to clipping. There was no statistically significant difference in poor outcome (modified Rankin Scale score $>2$ ) between these 2 treatment arms at any recorded time point during 6 years of follow-up. After the initial hospitalization, 1 of $241(0.4 \%)$ clipped saccular aneurysms and 21 of $115(18 \%)$ coiled saccular aneurysms required retreatment $(p<0.001)$. At the 6 -year follow-up, $95 \%$ $(95 / 100)$ of the clipped aneurysms were completely obliterated, compared with $40 \%(16 / 40)$ of the coiled aneurysms $(p<$ $0.001)$. There was no difference in morbidity between the 2 treatment groups $(p=0.10)$.

CONCLUSIONS In the subgroup of patients with saccular aneurysms enrolled in the BRAT, there was no significant difference between modified Rankin Scale outcomes at any follow-up time in patients with saccular aneurysms assigned to clipping compared with those assigned to coiling (intent-to-treat analysis). At the 6-year follow-up evaluation, rates of retreatment and complete aneurysm obliteration significantly favored patients who underwent clipping compared with those who underwent coiling.

Clinical trial registration no.: NCT01593267 (clinicaltrials.gov)

https://thejns.org/doi/abs/10.3171/2016.9.JNS161301

KEY WORDS BRAT; clip occlusion; coil embolization; intracranial aneurysm; ISAT; randomized trial; saccular aneurysms; SAH; subarachnoid hemorrhage; vascular disorders

$\mathrm{T}$ He 6-year results of the Barrow Ruptured Aneurysm Trial (BRAT) have been presented previously. ${ }^{11}$ This trial used a prospective intent-to-treat design that randomized all patients who were admitted with a diagnosis of subarachnoid hemorrhage $(\mathrm{SAH})$ to either a coiling or a clipping cohort. The design of the BRAT thus included patients with various types of aneurysms as well as patients who presented with a nonaneurysmal SAH. This all-inclusive patient database provides a unique opportunity to assess the frequency and treatment results of the various causes of nontraumatic SAH. Because all previous prospective trials comparing clipping and coiling are

ABBREVIATIONS AVM = arteriovenous malformation; BRAT = Barrow Ruptured Aneurysm Trial; CARAT = Cerebral Aneurysm Rerupture After Treatment; ISAT = International Subarachnoid Aneurysm Trial; $\mathrm{mRS}=$ modified Rankin Scale; $\mathrm{SAH}=$ subarachnoid hemorrhage.

SUBMITTED May 20, 2016. ACCEPTED September 7, 2016.

INCLUDE WHEN CITING Published online February 24, 2017; DOI: 10.3171/2016.9.JNS161301. 
based on cohorts restricted to saccular aneurysms, we are presenting a detailed post hoc analysis of this specific cohort based on data from the most recent BRAT follow-up.

\section{Methods \\ Patient Population}

The study protocol for the BRAT was approved by the Institutional Review Board of St. Joseph's Hospital and Medical Center, Phoenix, Arizona, on November 12, 2002. The trial is registered at ClinicalTrials.gov (NCT01593267). A detailed description of the study protocol was provided in the report of the 1-year follow-up results. ${ }^{6}$ Briefly, all eligible patients with nontraumatic SAH who were admitted between March 2003 and January 2007 were included if permission was obtained. Of 500 eligible patients, 29 were excluded because consent was obtained in error from 28 and 1 patient rescinded consent, leaving 471 patients who were randomly assigned to treatment: 238 to the intent-to-clip cohort and 233 to the intent-to-coil cohort.

Because there were no anatomical exclusion criteria, patients with nonaneurysmal SAHs were also included, creating 6 categories of nontraumatic SAH: nonaneurysmal SAHs, aneurysms associated with arteriovenous malformations (AVMs) or fistulas, fusiform aneurysms, blister aneurysms, dissecting aneurysms, and saccular aneurysms.

Sixty-three patients received no treatment -3 patients in each cohort died before treatment was initiated, and 57 patients had nonaneurysmal SAHs. Although our 1-year outcome data included these untreated patients, they were excluded from analysis in the 3-year and 6-year publications because these outcomes were not associated with any treatment decision, and the outcomes in this nontreated group were identical between the 2 assigned cohorts. ${ }^{6,10,11}$ Of 408 patients who received treatment, 209 were randomized to clipping and 199 to coiling.

After a patient was assigned to coiling or clipping, the treating surgeon determined whether that patient was better served by receiving the assigned treatment or by crossing over to the alternative treatment. Of the 209 patients assigned to clipping, 4 were crossed over to coiling (crossover rate $1.9 \%$ ). Of the 199 patients assigned to coiling, 75 were crossed over to clipping (crossover rate 38\%). Hematomas were the reason for crossover in 14 (19\%) of the 75 patients who crossed over from coiling to clipping. The remaining patients were crossed over due to anatomical features that the endovascular physician treating them believed would not be amenable to coiling.

Aneurysm type was determined from angiographic studies, surgical inspection, and endovascular treatment. A substantial degree of arbitrary designation of aneurysm type occurs because the differences are not always clear. Thus, the highest fidelity was ensured by submitting all nonsaccular aneurysms for further review by nontreating physicians. The extent of aneurysm obliteration was adjudicated by an independent neuroradiologist not involved in treatment. Although some aneurysms were reclassified, statistical analysis revealed no significant difference between the initial and final adjudication. The final designa-
TABLE 1. Causes of nontraumatic SAH

\begin{tabular}{lcc}
\hline \multicolumn{1}{c}{ Type } & No. $(\mathrm{N}=471)$ & $\%^{*}$ \\
\hline Aneurysms associated w/ AVM or fistula & 3 & 1 \\
\hline Fusiform aneurysms & 5 & 1 \\
\hline Blister aneurysms & 8 & 2 \\
\hline Dissecting aneurysms & 36 & 8 \\
\hline Nonaneurysmal SAH & 57 & 12 \\
\hline Saccular aneurysms & 362 & 77 \\
\hline
\end{tabular}

* Percentages total $>100 \%$ due to rounding.

tion of aneurysm type for each lesion was used for this study.

\section{Outcome Analysis}

A research nurse collected the follow-up data and performed the modified Rankin Scale (mRS) examinations. Outcomes were recorded at 6 months and at 1,3 , and 6 years, with the final follow-up to be performed at 10 years. Adverse events were recorded as they occurred. The primary outcome was based on $\mathrm{mRS}$ scores, which were analyzed on an intent-to-treat basis by the assigned treatment group. As previously reported, the 2 groups were well matched, with the exception of aneurysm location in the posterior circulation. ${ }^{6,10,11}$

Additional data analyses included aneurysm type and outcome based on actual treatment, outcome of crossedover patients, rebleeding, death, aneurysm size, aneurysm location, and extent of aneurysm obliteration as assessed on imaging studies by a neuroradiologist not involved in treatment (R.C.W.). This report concentrates on the analyses of outcome only for patients with saccular aneurysms.

\section{Results}

Causes of nontraumatic SAH in the 471 patients were classified as nonaneurysmal SAH $(12 \% ; 57 / 471)$ and aneurysmal SAH, with the latter group including aneurysms associated with AVMs or fistulas and fusiform, blister, dissecting, and saccular aneurysms (Table 1). Saccular aneurysms represented $77 \%$ (362/471) of all nontraumatic SAHs. The next largest group was the dissecting aneurysm group, which represented 8\% (36/471) of all nontraumatic SAHs and 9\% (36/414) of all aneurysmal SAHs. Blister aneurysm represented $2 \%(8 / 471)$ of nontraumatic SAHs, and fusiform aneurysms (5/471) and aneurysms associated with AVMs or fistulas (3/471) represented 1\% each.

When the analysis was restricted to aneurysms, excluding the nonaneurysmal SAH group, 414 patients remained, of whom $87 \%$ (362/414) had saccular aneurysms. The aneurysm types and their assignment to coiling or clipping are summarized in Table 2. For the 362 patients with saccular aneurysms enrolled in the BRAT, randomization resulted in equal assignments of 181 patients to each of the 2 intent-to-treat cohorts. In addition, the critical Hunt and Hess scores demonstrated good parity between the 2 assigned cohorts (Table 3).

In each cohort, 3 patients died before treatment and 178 were treated. Of the 178 clip-assigned patients with sac- 
TABLE 2. Distribution of aneurysm types by assigned treatment*

\begin{tabular}{|c|c|c|c|c|c|c|}
\hline \multirow{2}{*}{$\begin{array}{c}\text { Type of } \\
\text { Aneurysm }\end{array}$} & \multicolumn{2}{|c|}{$\begin{array}{c}\text { Total Assigned } \\
(n=414)\end{array}$} & \multicolumn{2}{|c|}{$\begin{array}{c}\text { Assigned to } \\
\text { Coiling }(n=202)\end{array}$} & \multicolumn{2}{|c|}{$\begin{array}{c}\text { Assigned to } \\
\text { Clipping }(n=212)\end{array}$} \\
\hline & No. & $\%$ & No. & $\%$ & No. & $\%$ \\
\hline Saccular & 362 & 87 & 181 & 44 & 181 & 44 \\
\hline Anterior & 319 & 77 & 160 & 38 & 159 & 38 \\
\hline Posterior & 43 & 10 & 21 & 5 & 22 & 5 \\
\hline Dissecting & 36 & 9 & 16 & 4 & 20 & 5 \\
\hline Blister & 8 & 2 & 3 & 1 & 5 & 1 \\
\hline Fusiform & 5 & 1 & 2 & $<1$ & 3 & 1 \\
\hline $\begin{array}{l}\text { AVM or } \\
\text { fistula }\end{array}$ & 3 & 1 & 0 & 0 & 3 & 1 \\
\hline
\end{tabular}

* Percentages for the subgroups were also calculated based on the total group of 414 patients.

cular aneurysms, $1(<1 \%)$ was crossed over to coiling, and $64(36 \%)$ of the 178 coil-assigned patients were crossed over to clipping (Fig. 1).

\section{Intent-to-Treat Analysis}

When the intent-to-treat analysis of clinical outcome was limited to patients with saccular aneurysms, no significant difference in $\mathrm{mRS}$ score $>2$ was found between the 2 treatment arms at any time point during the planned follow-up from hospital discharge to 6 years posttreatment (Table 4). This lack of difference in outcome also held true whether the categorized or uncategorized mRS scores were analyzed.

\section{Crossover Analysis}

Although a significant difference was found in clinical outcome between the 2 cohorts that actually received their assigned treatment (coil-coil and clip-clip) at 6 months and 1 year ( $\mathrm{p}=0.016$ and $\mathrm{p}=0.048$, respectively), this finding reflects the difference in crossover $(36 \%$ and $<1 \%$, respectively). The difference was no longer significant at 3 and 6 years ( $p=0.28$ and $p=0.16$, respectively). The analysis of clinical outcome by actual treatment for patients with saccular aneurysms is presented in Table 5 .

Following the initial hospitalization, 1 of 241 clipped saccular aneurysms and 21 of 115 coiled saccular aneurysms required retreatment $(\mathrm{p}<0.001)$ (Table 6). At the 6-year follow-up examination, 95\% (95/100) of the clipped aneurysms compared with $40 \%$ (16/40) of the coiled aneurysms were completely obliterated $(\mathrm{p}<0.001)$ (Table 7$)$. There was no difference in morbidity between the 2 treatment groups $(\mathrm{p}=0.10)$.

\section{Discussion}

In all other published prospective randomized trials ${ }^{4-6,9}$ comparing clipping and coiling, equipoise was required before randomization. In contrast, the BRAT was an intent-to-treat trial that entered and randomized all patients with nontraumatic SAH before the source of the hemorrhage was determined. The strength of the BRAT lies in its ability to provide unique information as to the cause
TABLE 3. Preoperative Hunt and Hess grade by assigned treatment in patients with saccular aneurysms

\begin{tabular}{|c|c|c|c|c|c|}
\hline \multirow{3}{*}{$\begin{array}{c}\text { Hunt \& } \\
\text { Hess } \\
\text { Grade }\end{array}$} & \multicolumn{4}{|c|}{ Assigned Treatment } & \multirow{3}{*}{$\begin{array}{c}p \\
\text { Valuef }\end{array}$} \\
\hline & \multicolumn{2}{|c|}{ Coiling $(n=181)$} & \multicolumn{2}{|c|}{ Clipping $(n=181)$} & \\
\hline & No. & $\%^{*}$ & No. & $\% \dagger$ & \\
\hline 1 & 20 & 11 & 21 & 12 & \\
\hline 2 & 68 & 38 & 71 & 39 & \\
\hline 3 & 50 & 28 & 53 & 29 & \\
\hline 4 & 30 & 17 & 25 & 14 & \\
\hline 5 & 12 & 7 & 9 & 5 & 0.47 \\
\hline
\end{tabular}

\footnotetext{
* Percentages total $>100 \%$ due to rounding.

$\dagger$ Percentages total $<100 \%$ due to rounding.

$\ddagger$ According to the Wilcoxon rank-sum test.
}

and prevalence of nontraumatic SAH and the ability of the 2 cohorts to actually undergo the assigned treatment, thus giving information regarding the frequency of crossovers. However, this attribute also results in an inherent weakness in the intent-to-treat design of the BRAT, in that a significant percentage of patients were randomized who had no aneurysm and thus required no treatment. In addition, aneurysms other than saccular types were included whose management was varied and difficult to analyze. Aneurysms with associated large hemorrhages or unique vascular anatomy perceived to be inappropriate for coiling were also randomized, thus accounting for a smaller percentage of patients for whom the assigned treatment was considered appropriate. Finally, the study protocol for the BRAT did not include an anticipated analysis restricted to saccular aneurysms. However, in light of the fact that all other randomized trials comparing clipping and coil-

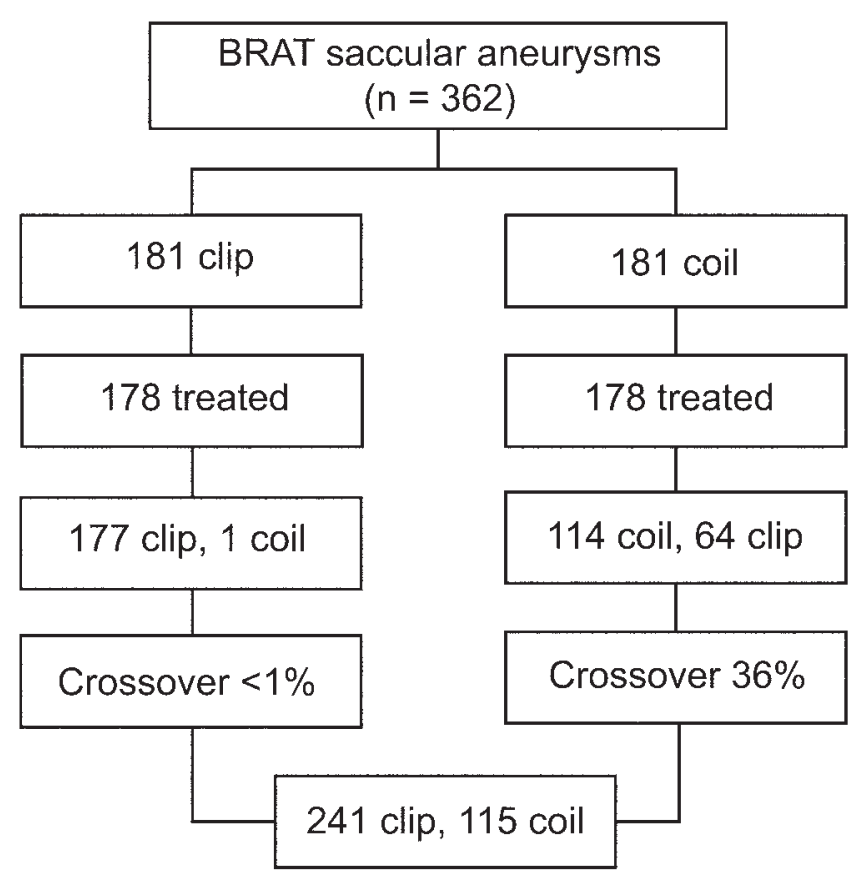

FIG. 1. Summary of cohort assignment, actual treatment, and percent crossover for 362 saccular aneurysms in the BRAT. 
TABLE 4. Outcome in patients with saccular aneurysms and $m R S$ scores $>2$ across BRAT follow-up by assigned treatment

\begin{tabular}{|c|c|c|c|c|c|c|c|c|}
\hline \multirow{2}{*}{$\begin{array}{l}\text { Time of } \\
\text { Follow-Up }\end{array}$} & \multirow{2}{*}{$\begin{array}{l}\text { No. of Pts Available } \\
\text { for Analysis }\end{array}$} & \multicolumn{2}{|c|}{ Coil-Assigned $(n=181)$} & \multicolumn{2}{|c|}{ Clip-Assigned $(n=181)$} & \multirow[b]{2}{*}{ OR } & \multirow{2}{*}{$\begin{array}{c}95 \% \\
\mathrm{Cl}\end{array}$} & \multirow{2}{*}{$\begin{array}{c}p \\
\text { Value* }\end{array}$} \\
\hline & & No. & $\%$ & No. & $\%$ & & & \\
\hline Randomization & 362 & & & & & & & \\
\hline At discharge & 360 & $119 / 180$ & 66 & $122 / 180$ & 68 & 1.08 & $0.70,1.67$ & 0.74 \\
\hline $6 \mathrm{mos}$ & 306 & $42 / 159$ & 26 & $49 / 147$ & 33 & 1.39 & $0.85,2.28$ & 0.19 \\
\hline $1 \mathrm{yr}$ & 317 & $43 / 158$ & 27 & $52 / 159$ & 33 & 1.30 & $0.80,2.11$ & 0.29 \\
\hline 3 yrs & 307 & $52 / 154$ & 34 & $50 / 153$ & 33 & 0.95 & $0.59,1.53$ & 0.84 \\
\hline 3 yrs; CF† & 324 & $52 / 162$ & 32 & $50 / 162$ & 31 & 0.94 & $0.59,1.51$ & 0.81 \\
\hline 6 yrs & 297 & $56 / 148$ & 38 & $59 / 149$ & 40 & 1.08 & $0.68,1.72$ & 0.76 \\
\hline 6 yrs; CF $\ddagger$ & 328 & $59 / 164$ & 36 & $59 / 164$ & 36 & 1.00 & $0.64,1.57$ & $>0.99$ \\
\hline
\end{tabular}

ing are limited to saccular aneurysms, it is appropriate to analyze this subgroup in the BRAT.

Of the 4 randomized trials $s^{4,5,9,11}$ in the literature that compare coiling and clipping, only the International Subarachnoid Aneurysm Trial (ISAT) has demonstrated the superiority of endovascular coiling over surgical clipping beyond the 1st year. Although the remaining 3 studies $^{4,5,11}$ were statistically underpowered, it is noteworthy that the larger 2 of the 3 (i.e., the BRAT ${ }^{11}$ and the study by $\mathrm{Li}$ et $\mathrm{al} .^{5}$ ) both showed 1-year trends of a magnitude similar to the original ISAT ${ }^{9}$ results favoring endovascular treatment. In this analysis of saccular aneurysms in the BRAT, there were $5.5 \%(\mathrm{p}=0.29)$ fewer poor outcomes in the coiling group at 1 year, whereas in the study by $\mathrm{Li}$ et al. there were $9.4 \%$ fewer dead or disabled patients in the endovascular treatment group. Again, these rates were similar in absolute magnitude to those in the ISAT, but they were not statistically significant because of the much smaller sample sizes.

However, during follow-up in the ISAT, there was a statistically significant increase in SAHs for aneurysms that were coiled compared with those that were clipped. ${ }^{8,9}$ Incomplete obliteration in the coiled cohort would reasonably explain this increase in SAHs.

In this report, we reanalyzed the BRAT results by the type of aneurysm responsible for SAH. Restricting the analysis to saccular aneurysms allows a meaningful comparison of the BRAT data with data from other studies whose entry criteria were restricted to saccular aneurysms. It also provides data on the frequency of other causes of nontraumatic SAH.

As previously described, a total of 471 patients with nontraumatic SAH were enrolled in the BRAT. ${ }^{6}$ As in other reports in the literature, this population included a small proportion $(n=57,12 \%)$ of patients with diagnostically negative SAH. The relatively benign history of this subgroup of patients has been well documented in the literature. ${ }^{1}$ Although these patients were initially randomized to clipping or coiling, they never received treatment and are not considered further in this analysis.

TABLE 5. Outcome in patients with saccular aneurysms and mRS scores $>2$ across BRAT follow-up by actual treatment $(n=356)$, and statistical comparison between groups*

\begin{tabular}{|c|c|c|c|c|c|c|c|c|c|}
\hline \multirow{2}{*}{$\begin{array}{l}\text { Time of } \\
\text { Follow-Up }\end{array}$} & \multirow{2}{*}{$\begin{array}{c}\text { No. of Pts Available } \\
\text { for Analysis }\end{array}$} & \multicolumn{2}{|c|}{ Coil-Coil $(n=114)$} & \multicolumn{2}{|c|}{ Coil-Clip $(n=64)$} & \multicolumn{2}{|c|}{ Clip-Clip $(n=177)$} & \multicolumn{2}{|c|}{ Clip-Coil $(n=1)$} \\
\hline & & No. & $\%$ & No. & $\%$ & No. & $\%$ & No. & $\%$ \\
\hline At discharge & $354 \dagger$ & $65 / 114$ & 57 & $51 / 63$ & 81 & $118 / 176$ & 67 & $1 / 1$ & 100 \\
\hline $6 \mathrm{mos}$ & 300 & $18 / 101$ & 18 & $21 / 55$ & 38 & $45 / 143$ & 31 & $1 / 1$ & 100 \\
\hline $1 \mathrm{yr}$ & 311 & $20 / 101$ & 20 & $20 / 54$ & 37 & $48 / 155$ & 31 & $1 / 1$ & 100 \\
\hline 3 yrs & 301 & $24 / 98$ & 24 & $25 / 53$ & 47 & $46 / 149$ & 31 & $1 / 1$ & 100 \\
\hline 3 yrs w/ CF & 318 & $24 / 103$ & 23 & $25 / 56$ & 45 & $46 / 158$ & 29 & $1 / 1$ & 100 \\
\hline 6 yrs & 291 & $27 / 93$ & 29 & $26 / 52$ & 50 & $55 / 145$ & 38 & $1 / 1$ & 100 \\
\hline 6 yrs w/ CF§ & 322 & $28 / 104$ & 27 & $28 / 57$ & 49 & $55 / 160$ & 34 & $1 / 1$ & 100 \\
\hline
\end{tabular}

* The following comparisons were made: coil-coil and coil-clip $(p=0.005$ at 3 years, $p=0.01$ at 6 years); coil-clip and clip-clip ( $p=0.03$ at 3 years, $p=0.13$ at 6 years); and coil-coil and clip-clip ( $p=0.28$ at 3 years, $p=0.16$ at 6 years).

$\dagger$ Two of the 356 patients who were treated died before discharge.

$\mp$ Includes patients seen at 1 year but not at 3 years.

$\S$ Includes patients seen at 1 and 3 years but not at 6 years. 
TABLE 6. Retreatments of saccular aneurysms at 6 years after discharge, categorized by original (actual) treatment type

\begin{tabular}{ccccc}
\hline $\begin{array}{c}\text { Original } \\
\text { Treatment Type }\end{array}$ & $\begin{array}{c}\text { No. of } \\
\text { Patients }\end{array}$ & $\begin{array}{c}\text { No. of } \\
\text { Retreatments }\end{array}$ & $\%$ & $\begin{array}{c}p \\
\text { Value }\end{array}$ \\
\cline { 1 - 4 } Coiling & 115 & 21 & 18 & $<0.001$ \\
\cline { 1 - 4 } Clipping & 241 & 1 & 0.4 & \\
\hline
\end{tabular}

The sources of SAH in the remaining 414 patients are summarized in Table 2. Saccular aneurysms made up $87 \%$ (362) of all cases of aneurysmal SAHs in this group. The remaining aneurysm types were of such small numbers and were treated with such a variety of methods as to preclude any substantive comparisons. Of the 356 treated saccular aneurysms, 178 were randomized to the intent-tocoil cohort, and 178 were randomized to the intent-to-clip cohort.

As described in previous reports of the BRAT, the risk of a poor outcome for all aneurysm types (defined as $\mathrm{mRS}$ score $>2$ ) was significantly lower at 1 year in patients assigned to endovascular treatment but was not statistically significant at 3- or 6-year follow-up. ${ }^{6,10,11}$ When the outcome analysis was restricted to saccular aneurysms, no difference was found in patients with an $\mathrm{mRS}$ score $>2$ between the 2 assigned cohorts at any recorded time point from discharge through the 6-year follow-up evaluation (Table 4). Although the absolute differences favoring endovascular treatment at 6 months and 1 year $(6.9 \%$ and $5.5 \%$, respectively) are virtually identical to those in the ISAT, the much smaller sample size in the BRAT limits its statistical power.

Of the 178 patients in the intent-to-coil cohort, 114 (64\%) received their designated treatment, and 64 (36\%) were crossed over to clipping for various reasons. In the intent-to-clip cohort, all but 1 patient received the designated treatment, with the 1 exception being a patient who was crossed over to coiling. Not unexpectedly, the outcomes of the patients who crossed over from coiling to clipping, which included all the patients with large intraparenchymal hemorrhages, were worse than the outcomes of patients in the coil-coil group, which explains the better actual treatment results in the coil-coil group at 6 months and 1 year $(p=0.016$ and $p=0.048$, respectively). However, no significant difference was found at 3 and 6 years $(p=0.28$ and $p=0.16$, respectively).

Incomplete obliteration of aneurysms and major recurrence requiring retreatment after coil embolization are well documented in the endovascular literature. A recent meta-analysis of recurrence rates identified aneurysm recurrence in $20.8 \%$ of cases (95\% CI 19.8\%-21.9\%), with retreatment needed in $10.3 \%$ (95\% CI 9.5\%-11.0\%). ${ }^{2}$ In the present analysis, obliteration was complete in $96 \%$ of the clipped aneurysms compared with $48 \%$ of the coiled aneurysms $(p<0.001)$ at the 6-year follow-up. After the initial hospitalization, 21 of 115 coiled saccular aneurysms required retreatment compared with 1 of 241 clipped aneurysms $(\mathrm{p}<0.001)$.

Incomplete obliteration and recurrence are associated with an increased risk of rebleeding. The Cerebral Aneu-
TABLE 7. Complete aneurysm obliteration categorized by actual treatment

\begin{tabular}{cccc}
\hline $\begin{array}{c}\text { Time of } \\
\text { Follow-Up }\end{array}$ & $\begin{array}{c}\text { Coiling No./ } \\
\text { Total (\%) }\end{array}$ & $\begin{array}{c}\text { Clipping No.I } \\
\text { Total (\%) }\end{array}$ & p Value \\
\hline Postop & $60 / 115(52)$ & $205 / 236(87)$ & $<0.001$ \\
\hline 3 yrs & $27 / 60(45)$ & $104 / 118(88)$ & $<0.001$ \\
\hline 6 yrs & $16 / 40(40)$ & $95 / 100(95)$ & $<0.001$ \\
\hline
\end{tabular}

rysm Rerupture After Treatment (CARAT) study showed that in 1001 patients treated at 9 high-volume centers between 1996 and 2005, the risk for rebleeding was strongly associated with the degree of aneurysmal occlusion (cumulative risk: $1.1 \%$ for complete occlusion, $2.9 \%$ for $91 \%$ $99 \%$ occlusion, $5.9 \%$ for $70 \%-90 \%$ occlusion, and $17.6 \%$ for $<70 \%$ occlusion). ${ }^{3}$ Thus, the CARAT study demonstrated that protection against rebleeding was related to the quality of the aneurysm occlusion, and it emphasized the importance of long-term follow-up. The BRAT is an ongoing study, with plans to continue follow-up out to 10 years in all patients.

The current study has several limitations. It is a post hoc review, with the assignment of aneurysm types to the various categories being somewhat subjective.

\section{Conclusions}

The present analysis of the BRAT provides good data for establishing the causes and prevalence of nontraumatic SAH. Although this study had insufficient power to detect differences of the magnitude noted in the ISAT, no statistically significant difference in mRS scores was noted between the 2 treatment groups. At 6-year follow-up, retreatment and complete aneurysm obliteration significantly favored patients who underwent clipping compared with coiling. Clearly, these results provide sound evidence in support of a new randomized trial, preferably an intent-totreat trial that will help determine the appropriate treatment for patients with an SAH.

\section{Acknowledgments}

Funding for this manuscript came from the Barrow Neurological Foundation and the Hanley Aneurysm Fund.

\section{References}

1. Elhadi AM, Zabramski JM, Almefty KK, Mendes GA, Nakaji P, McDougall CG, et al: Spontaneous subarachnoid hemorrhage of unknown origin: hospital course and longterm clinical and angiographic follow-up. J Neurosurg 122:663-670, 2015

2. Ferns SP, Sprengers ME, van Rooij WJ, Rinkel GJ, van Rijn JC, Bipat S, et al: Coiling of intracranial aneurysms: a systematic review on initial occlusion and reopening and retreatment rates. Stroke 40:e523-e529, 2009

3. Johnston SC, Dowd CF, Higashida RT, Lawton MT, Duckwiler GR, Gress DR: Predictors of rehemorrhage after treatment of ruptured intracranial aneurysms: the Cerebral Aneurysm Rerupture After Treatment (CARAT) study. Stroke 39:120-125, 2008

4. Koivisto T, Vanninen R, Hurskainen H, Saari T, Hernesniemi 
J, Vapalahti M: Outcomes of early endovascular versus surgical treatment of ruptured cerebral aneurysms. A prospective randomized study. Stroke 31:2369-2377, 2000

5. Li ZQ, Wang QH, Chen G, Quan Z: Outcomes of endovascular coiling versus surgical clipping in the treatment of ruptured intracranial aneurysms. J Int Med Res 40:2145-2151, 2012

6. McDougall CG, Spetzler RF, Zabramski JM, Partovi S, Hills NK, Nakaji P, et al: The Barrow Ruptured Aneurysm Trial. J Neurosurg 116:135-144, 2012

7. Molyneux AJ, Birks J, Clarke A, Sneade M, Kerr RS: The durability of endovascular coiling versus neurosurgical clipping of ruptured cerebral aneurysms: 18 year follow-up of the UK cohort of the International Subarachnoid Aneurysm Trial (ISAT). Lancet 385:691-697, 2015

8. Molyneux AJ, Kerr RS, Birks J, Ramzi N, Yarnold J, Sneade M, et al: Risk of recurrent subarachnoid haemorrhage, death, or dependence and standardised mortality ratios after clipping or coiling of an intracranial aneurysm in the International Subarachnoid Aneurysm Trial (ISAT): long-term follow-up. Lancet Neurol 8:427-433, 2009

9. Molyneux AJ, Kerr RS, Yu LM, Clarke M, Sneade M, Yarnold JA, et al: International subarachnoid aneurysm trial (ISAT) of neurosurgical clipping versus endovascular coiling in 2143 patients with ruptured intracranial aneurysms: a randomised comparison of effects on survival, dependency, seizures, rebleeding, subgroups, and aneurysm occlusion. Lancet 366:809-817, 2005

10. Spetzler RF, McDougall CG, Albuquerque FC, Zabramski JM, Hills NK, Partovi S, et al: The Barrow Ruptured Aneurysm Trial: 3-year results. J Neurosurg 119:146-157, 2013

11. Spetzler RF, McDougall CG, Zabramski JM, Albuquerque FC, Hills NK, Russin JJ, et al: The Barrow Ruptured Aneurysm Trial: 6-year results. J Neurosurg 123:609-617, 2015

\section{Disclosures}

Dr. McDougall reports being a consultant for Covidien and MicroVention, Inc.

\section{Author Contributions}

Conception and design: Spetzler. Analysis and interpretation of data: Spetzler. Drafting the article: all authors. Critically revising the article: all authors. Reviewed submitted version of manuscript: all authors.

\section{Correspondence}

Robert F. Spetzler, c/o Neuroscience Publications, Barrow Neurological Institute, St. Joseph's Hospital and Medical Center, 350 W Thomas Rd., Phoenix, AZ 85013. email: neuropub@ dignityhealth.org. 